\title{
A Computational Quadruple Laplace Transform for the Solution of Partial Differential Equations
}

\author{
Hamood Ur Rehman', Muzammal Iftikhar'1, Shoaib Saleem¹, Muhammad Younis², \\ Abdul Mueed ${ }^{3}$ \\ ${ }^{1}$ Department of Mathematics, University of Education, Okara Campus, Okara, Pakistan \\ ${ }^{2}$ Center for Undergraduate Studies, University of the Punjab, Lahore, Pakistan \\ ${ }^{3}$ Air University Multan Campus, Multan, Pakistan \\ Email: hamood84@gmail.com, muzamil.iftikhar@ue.edu.pk, shaby455@yahoo.com, younis.pu@gmail.com, \\ abdulmueed3@hotmail.com
}

Received 11 October 2014; revised 2 November 2014; accepted 16 November 2014

Copyright (C) 2014 by authors and Scientific Research Publishing Inc.

This work is licensed under the Creative Commons Attribution International License (CC BY).

http://creativecommons.org/licenses/by/4.0/

(c) (i) Open Access

\begin{abstract}
In this paper, we proposed new results in quadruple Laplace transform and proved some properties concerned with quadruple Laplace transform. We also developed some applications based on these results and solved homogeneous as well as non-homogeneous partial differential equations involving four variables. The performance of quadruple Laplace transform is shown to be very encouraging by concrete examples. An elementary table of quadruple Laplace transform is also provided.
\end{abstract}

Keywords

Quadruple Laplace Transform, Exact Solution, Convolution, Partial Differential Equation, Homogeneous and Non-Homogeneous Problems

\section{Introduction}

Many engineering and science fields encounter linear or non-linear partial differential equations describing the physical phenomena. A number of methods (for example, approximate and exact methods) can be used to determine the solutions of differential equations. Mostly, it may be complicated to solve these equations analytically. Such equations are commonly solved by integral transforms such as Laplace and Fourier transforms and the worth of Laplace and Fourier transforms lies in their ability to transform differential equations into algebraic 
equations, which allows a systematic and simple way to find solution. The numerical methods can provide approximate solutions rather than analytic solutions of the problems [1]. A number of aspects of these methods have been studied in [2] [3].

The Laplace transform has been effectively used to solve linear and non-linear ordinary and partial differential equations and is used extensively in electrical engineering. The Laplace transform reduces a linear differential equation to an algebraic equation, which can be solved by rules of algebra. The original differential equation can then be solved by applying the inverse Laplace transform. The Heaviside first proposed a scheme, without using the Laplace transform (see [4] and references therein).

Eltayeb and Kiliçman [5] applied double Laplace transform to find the solution of general linear telegraph and partial integro-differential equations. Dahiya and Najafai [6] established new theorems for calculating the Laplace transforms of $n$-dimensions and application of these theorems to a number of commonly used special functions was considered, and in the end, authors solved one-dimensional wave equation involving special functions using two dimensional Laplace transforms. Aghili and Moghaddam [7] presented a new theorem and corollary on multi-dimensional Laplace transformations. Authors further developed some applications based on these results. Kiliçman and Eltayeb [8] discussed the relationship between Sumudu and Laplace transforms and further made some comparison on the solutions. Authors provided some counter examples. Cheniguel and Reghioua [9] investigated the solution of three-dimensional diffusion equation with non-local condition using Adomian decomposition method. Atangana [4] introduced the triple Laplace transform. Author discussed some properties and theorems about the triple Laplace transform. Moreover, author used the operator to solve some kind of third-order differential equation.

The aim of this paper is to discuss some properties and theorems about the quadruple Laplace transform and give a good strategy for solving the fourth order partial differential equations in engineering and physics fields, by quadruple Laplace transform.

First of all, we recall the following definitions.

The double Laplace transform of a continuous function $f(x, y)$ can be defined [5] [10] as

$$
F^{x y}(p, q)=L_{x y} f(x, y)=\iint_{0}^{\infty} \mathrm{e}^{-p x} \mathrm{e}^{-q y} f(x, y) \mathrm{d} x \mathrm{~d} y,
$$

where $x, y>0$ and $p, q$ are Laplace variables.

The inverse double Laplace transform is defined as

$$
f(x, y)=L_{x y}^{-1} F^{x y}(p, q)=\frac{1}{2 \pi i} \int_{\alpha-i \infty}^{\alpha+i \infty} \mathrm{e}^{p x}\left(\frac{1}{2 \pi i} \int_{\beta-i \infty}^{\beta+i \infty} \mathrm{e}^{q y} F^{x y}(p, q) \mathrm{d} q\right) \mathrm{d} p .
$$

The triple Laplace transform [4] of a continuous function $f(x, y, z)$ can be defined as

$$
F^{x y z}(p, q, r)=L_{x y z} f(x, y, z)=\iiint_{0}^{\infty} \mathrm{e}^{-p x} \mathrm{e}^{-q y} \mathrm{e}^{-r z} f(x, y, z) \mathrm{d} x \mathrm{~d} y \mathrm{~d} z,
$$

where $x, y, z>0$ and $p, q, r$ are Laplace variables.

The inverse triple Laplace transform is defined as

$$
f(x, y, z)=L_{x y z}^{-1} F^{x y z}(p, q, r)=\frac{1}{2 \pi i} \int_{\alpha-i \infty}^{\alpha+i \infty} \mathrm{e}^{p x}\left(\frac{1}{2 \pi i} \int_{\beta-i \infty}^{\beta+i \infty} \mathrm{e}^{q y}\left(\frac{1}{2 \pi i} \int_{\xi-i \infty}^{\xi+i \infty} \mathrm{e}^{r z} F^{x y z}(p, q, r) \mathrm{d} r\right) \mathrm{d} q\right) \mathrm{d} p .
$$

In the following section definitions of quadruple Laplace transform, its inverse and some of its properties are presented.

\section{Definitions and Properties}

Before launching into the main part of the paper, we define some notations and terminologies which will remain standard.

$$
\begin{aligned}
& F^{w x y z}(p, q, r, s)=L_{w x y z} f(w, x, y, z), \\
& F^{w x y}(p, q, r, z)=L_{w x y} f(w, x, y, z), \\
& F^{w x}(p, q, y, z)=L_{w x} f(w, x, y, z), \\
& F^{w}(p, x, y, z)=L_{w} f(w, x, y, z) .
\end{aligned}
$$


Quadruple Laplace transform: Let $f$ be a continuous function of four variables, then, the quadruple Laplace transform of $f(w, x, y, z)$ is defined by

$$
F^{w x y z}(p, q, r, s)=L_{w x y z} f(w, x, y, z)=\iiint \int_{0}^{\infty} \mathrm{e}^{-p w} \mathrm{e}^{-q x} \mathrm{e}^{-r y} \mathrm{e}^{-s z} f(w, x, y, z) \mathrm{d} w \mathrm{~d} x \mathrm{~d} y \mathrm{~d} z,
$$

where $w, x, y, z>0$ and $p, q, r, s$ are Laplace variables.

It is to be noted that the quadruple Laplace transform operator is linear.

$$
L_{w x y z}\{a f(w, x, y, z)+b g(w, x, y, z)\}=a L_{w x y z}\{f(w, x, y, z)\}+b L_{w x y z}\{g(w, x, y, z)\} .
$$

Now, if the quadruple Laplace transform is known, its inverse is given by

$$
\begin{aligned}
f(w, x, y, t) & =L_{w x y z}^{-1} F^{w x y z}(p, q, r, s) \\
& =\frac{1}{2 \pi i} \int_{\alpha-i \infty}^{\alpha+i \infty} \mathrm{e}^{p w}\left(\frac{1}{2 \pi i} \int_{\beta-i \infty}^{\beta+\infty \infty} \mathrm{e}^{q x}\left(\frac{1}{2 \pi i} \int_{\gamma-i \infty}^{\gamma+i \infty} \mathrm{e}^{r y}\left(\frac{1}{2 \pi i} \int_{\delta-i \infty}^{\delta+i \infty} \mathrm{e}^{s z} F^{w x y z}(p, q, r, s) \mathrm{d} z\right) \mathrm{d} y\right) \mathrm{d} x\right) \mathrm{d} w .
\end{aligned}
$$

Quadruple Laplace transform for some partial derivatives of function of four variables are given as

1) Quadruple Laplace transform for first order partial derivative of function of four variables

$$
L_{w x y z}\left[\frac{\partial}{\partial z} f(w, x, y, z)\right]=s F^{w x y z}(p, q, r, s)-F^{w x y}(p, q, r, 0) .
$$

2) Quadruple Laplace transform for second order partial derivative of function of four variables

$$
\begin{aligned}
& L_{w x y z}\left[\frac{\partial^{2}}{\partial w^{2}} f(w, x, y, z)\right]=p^{2} F^{w x y z}(p, q, r, s)-p F^{x y z}(0, q, r, s)-\frac{\partial}{\partial w} F^{x y z}(0, q, r, s), \\
& L_{w x y z}\left[\frac{\partial^{2}}{\partial x^{2}} f(w, x, y, z)\right]=q^{2} F^{w x y z}(p, q, r, s)-q F^{w y z}(p, 0, r, s)-\frac{\partial}{\partial x} F^{w y z}(p, 0, r, s), \\
& L_{w x y z}\left[\frac{\partial^{2}}{\partial y^{2}} f(w, x, y, z)\right]=r^{2} F^{w x y z}(p, q, r, s)-r F^{w x z}(p, q, 0, s)-\frac{\partial}{\partial y} F^{w x z}(p, q, 0, s) .
\end{aligned}
$$

3) Quadruple Laplace transform for the mixed fourth order partial derivative of function of four variables

$$
\begin{aligned}
& L_{w x y z}\left[\frac{\partial^{4}}{\partial w \partial x \partial y \partial z} f(w, x, y, z)\right] \\
& =p q r s F^{w x y z}(p, q, r, s)-p q r F^{w x y}(p, q, r, 0)-p q s F^{w x z}(p, q, 0, s)-p r s F^{w y z}(p, 0, r, s) \\
& -q r s F^{x y z}(0, q, r, s)+p q F^{w x}(p, q, 0,0)+p r F^{w y}(p, 0, r, 0)+p s F^{w z}(p, 0,0, s) \\
& +q r F^{x y}(0, q, r, 0)+q s F^{x z}(0, q, 0, s)+r s F^{y z}(0,0, r, s)-p F^{w}(p, 0,0,0) \\
& -q F^{x}(0, q, 0,0)-r F^{y}(0,0, r, 0)-s F^{z}(0,0,0, s)+f(0,0,0,0) . \\
& L_{w w x x}\left[\frac{\partial^{4}}{\partial w^{2} \partial x^{2}} f(w, x, y, z)\right] \\
& =p^{2} q^{2} F^{w w x x}(p, q, y, z)-p^{2} q F^{x}(0, q, y, z)-p q^{2} F^{w}(p, 0, y, z) \\
& \quad+p q f(0,0, y, z)-p^{2} F_{w}^{w}(p, 0, y, z)-q^{2} F_{x}^{x}(0, q, y, z) \\
& +p f_{x}(p, 0, y, z)+q f_{w}(0, q, y, z)+f_{w x}(0,0,0,0) .
\end{aligned}
$$

The uniqueness and existence of the quadruple Laplace transform is discussed in the following section.

\section{Uniqueness and Existence of the Quadruple Laplace Transform}

Consider $f(w, x, y, z)$ be a continuous function on the interval $[0, \infty)$. Also, assume that $f(w, x, y, z)$ is of exponential order, that is, there exists some constants $a, b, c, d \in R$ such that $f(w, x, y, z)$ satisfy the fol- 
lowing condition

$$
\sup _{w, x, y, z>0}=\frac{|f(w, x, y, z)|}{\mathrm{e}^{a w+b x+c y+d z}}<\infty .
$$

The quadruple Laplace transform

$$
F^{w x y z}(p, q, r, s)=L_{w x y z} f(w, x, y, z)=\iiint \int_{0}^{\infty} \mathrm{e}^{-p w} \mathrm{e}^{-q x} \mathrm{e}^{-r y} \mathrm{e}^{-s z} \mathrm{~d} w \mathrm{~d} x \mathrm{~d} y \mathrm{~d} z,
$$

exists for all $p>a, q>b, r>c, s>d$ and satisfy the condition (3.1). The following theorem explains the uniqueness.

Theorem 1. Let $f(w, x, y, z)$ and $g(w, x, y, z)$ is defined be continuous functions defined for $w, x, y, z \geq 0$ and having Laplace transforms, $F^{\text {wxyz }}(p, q, r, s)$ and $G^{\text {wxyz }}(p, q, r, s)$ respectively. If $F^{w x y z}(p, q, r, s)=G^{w x y z}(p, q, r, s)$, then $f(w, x, y, z)=g(w, x, y, z)$.

Proof If $\alpha, \beta, \gamma, \delta$ are sufficiently large. Then, from the definition of the quadruple inverse Laplace transform, we have

$$
f(w, x, y, z)=\frac{1}{2 \pi i} \int_{\alpha-i \infty}^{\alpha+i \infty} \mathrm{e}^{p w}\left(\frac{1}{2 \pi i} \int_{\beta-i \infty}^{\beta+i \infty} \mathrm{e}^{q x}\left(\frac{1}{2 \pi i} \int_{\gamma-i \infty}^{\gamma+i \infty} \mathrm{e}^{r y}\left(\frac{1}{2 \pi i} \int_{\delta-i \infty}^{\delta+i \infty} \mathrm{e}^{s z} F^{w x y z}(p, q, r, s) \mathrm{d} z\right) \mathrm{d} y\right) \mathrm{d} x\right) \mathrm{d} w .
$$

Using the hypothesis, $F^{\text {wxyz }}(p, q, r, s)=G^{\text {wxy }}(p, q, r, s)$, the expression (3.3) can be written as

$$
\begin{aligned}
f(w, x, y, z) & =\frac{1}{2 \pi i} \int_{\alpha-i \infty}^{\alpha+i \infty} \mathrm{e}^{p w}\left(\frac{1}{2 \pi i} \int_{\beta-i \infty}^{\beta+i \infty} \mathrm{e}^{q x}\left(\frac{1}{2 \pi i} \int_{\gamma-i \infty}^{\gamma+i \infty} \mathrm{e}^{r y}\left(\frac{1}{2 \pi i} \int_{\delta-i \infty}^{\delta+i \infty} \mathrm{e}^{s z} G^{w x y z}(p, q, r, s) \mathrm{d} z\right) \mathrm{d} y\right) \mathrm{d} x\right) \mathrm{d} w \\
& =g(w, x, y, z),
\end{aligned}
$$

this completes the proof.

\section{Convolution Theorem for the Quadruple Laplace Transform}

In this section, we will give some definitions of convolution for functions and state the convolution theorem of the quadruple Laplace transform.

Consider the functions $f_{1}(w, x, y, z), f_{2}(w, x, y, z), f_{3}(w, x, y, z)$ and $f_{4}(w, x, y, z)$.

The convolution of functions $f_{1}$ and $f_{2}$ can be defined as

$$
f_{1} * f_{2}=\int_{0}^{w} \int_{0}^{x} \int_{0}^{y} \int_{0}^{z} f_{1}(w-\rho, x-\varphi, y-\psi, z-\eta) f_{2}(\rho, \varphi, \psi, \eta) \mathrm{d} \rho \mathrm{d} \varphi \mathrm{d} \psi \mathrm{d} \eta .
$$

The convolution of functions $f_{1}, f_{2}$ and $f_{3}$ can be defined as

$$
\begin{aligned}
f_{1} *\left(f_{2} * f_{3}\right)= & \int_{0}^{w} \int_{0}^{x} \int_{0}^{y} \int_{0}^{z} f_{1}\left(w-\left(w_{1}+\rho\right), x-\left(x_{1}+\varphi\right), y-\left(y_{1}+\psi\right), z\right. \\
& \left.-\left(z_{1}+\eta\right)\right) f_{2}\left(w_{1}-\rho, x_{1}-\varphi, y_{1}-\psi, z_{1}-\eta\right) f_{3}(\rho, \varphi, \psi, \eta) \mathrm{d} \rho \mathrm{d} \varphi \mathrm{d} \psi \mathrm{d} \eta .
\end{aligned}
$$

Similarly, the convolution of functions $f_{1}, f_{2}, f_{3}$ and $f_{4}$ can be defined as

$$
\begin{aligned}
f_{1} *\left(f_{2} *\left(f_{3} * f_{4}\right)\right)= & \int_{0}^{w} \int_{0}^{x} \int_{0}^{y} \int_{0}^{z} f_{1}\left(w-\left(w_{1}+w_{2}+\rho\right), x-\left(x_{1}+x_{2}+\varphi\right), y\right. \\
& \left.-\left(y_{1}+y_{2}+\psi\right), z-\left(z_{1}+z_{2}+\eta\right)\right) f_{2}\left(w_{1}-\left(w_{2}+\rho\right), x_{1}-\left(x_{2}+\varphi\right),\right. \\
& \left.-y_{1}-\left(y_{2}+\psi\right), z_{1}\left(z_{2}+\eta\right)\right) f_{3}\left(w_{2}-\rho, x_{2}-\varphi, y_{2}-\psi, z_{2}-\eta\right) \\
& -f_{4}(\rho, \varphi, \psi, \eta) \mathrm{d} \rho \mathrm{d} \varphi \mathrm{d} \psi \mathrm{d} \eta .
\end{aligned}
$$

Theorem 2. (Convolution Theorem) If

$$
\begin{aligned}
& F_{1}^{w x y z}(p, q, r, s)=\iiint \int_{0}^{\infty} \mathrm{e}^{-p w} \mathrm{e}^{-q x} \mathrm{e}^{-r y} \mathrm{e}^{-s z} f_{1}(w, x, y, z) \mathrm{d} w \mathrm{~d} x \mathrm{~d} y \mathrm{~d} z, \\
& F_{2}^{w x y z}(p, q, r, s)=\iiint \int_{0}^{\infty} \mathrm{e}^{-p w} \mathrm{e}^{-q x} \mathrm{e}^{-r y} \mathrm{e}^{-s z} f_{2}(w, x, y, z) \mathrm{d} w \mathrm{~d} x \mathrm{~d} y \mathrm{~d} z,
\end{aligned}
$$




$$
F_{3}^{w x y z}(p, q, r, s)=\iiint \int_{0}^{\infty} \mathrm{e}^{-p w} \mathrm{e}^{-q x} \mathrm{e}^{-r y} \mathrm{e}^{-s z} f_{3}(w, x, y, z) \mathrm{d} w \mathrm{~d} x \mathrm{~d} y \mathrm{~d} z,
$$

are convergent at the point $(p, q, r, s)$ and if

$$
F_{4}^{w x y z}(p, q, r, s)=\iiint \int_{0}^{\infty} \mathrm{e}^{-p w} \mathrm{e}^{-q x} \mathrm{e}^{-r y} \mathrm{e}^{-s z} f_{4}(w, x, y, z) \mathrm{d} w \mathrm{~d} x \mathrm{~d} y \mathrm{~d} z,
$$

is absolutely convergent, then, the following expression

$$
F^{w x y z}(p, q, r, s)=F_{1}^{w x y z}(p, q, r, s) F_{2}^{w x y z}(p, q, r, s) F_{3}^{w x y z}(p, q, r, s) F_{4}^{w x y z}(p, q, r, s),
$$

is the Laplace transform of the function

and the integral

$$
\begin{aligned}
f(w, x, y, z)= & \int_{0}^{w} \int_{0}^{x} \int_{0}^{y} \int_{0}^{z} f_{1}\left(w-\left(w_{1}+w_{2}+\rho\right), x-\left(x_{1}+x_{2}+\varphi\right), y\right. \\
& \left.-\left(y_{1}+y_{2}+\psi\right), z-\left(z_{1}+z_{2}+\eta\right)\right) f_{2}\left(w_{1}-\left(w_{2}+\rho\right), x_{1}-\left(x_{2}+\varphi\right),\right. \\
& \left.-y_{1}-\left(y_{2}+\psi\right), z_{1}\left(z_{2}+\eta\right)\right) f_{3}\left(w_{2}-\rho, x_{2}-\varphi, y_{2}-\psi, z_{2}-\eta\right) \\
& -f_{4}(\rho, \varphi, \psi, \eta) \mathrm{d} \rho \mathrm{d} \varphi \mathrm{d} \psi \mathrm{d} \eta,
\end{aligned}
$$

is convergent at the point $(p, q, r, s)$, see the proof in [11].

\section{Properties of Quadruple Laplace Transform}

In this sectioon, some properties of quadruple Laplace transform are presented

Property (1)

$$
F^{w x y z}(p+a, q+b, r+c, s+d)=L_{w x y z}\left[\mathrm{e}^{-a w} \mathrm{e}^{-b x} \mathrm{e}^{-c y} \mathrm{e}^{-d z} f(w, x, y, z)\right](p, q, r, s) .
$$

Proof: By definition (2.1) of quadruple Laplace transform left hand side of (5.1) can be solved as

$$
\begin{aligned}
& L_{w x y z}\left[\mathrm{e}^{-a w} \mathrm{e}^{-b x} \mathrm{e}^{-c y} \mathrm{e}^{-d z} f(w, x, y, z)\right](p, q, r, s) \\
& =\iiint \int_{0}^{\infty} \mathrm{e}^{-a w} \mathrm{e}^{-b x} \mathrm{e}^{-c y} \mathrm{e}^{-d z} \mathrm{e}^{-p w} \mathrm{e}^{-q x} \mathrm{e}^{-r y} \mathrm{e}^{-s z} f(w, x, y, z) \mathrm{d} w \mathrm{~d} x \mathrm{~d} y \mathrm{~d} z \\
& =\iiint_{0}^{\infty} \mathrm{e}^{-a w} \mathrm{e}^{-b x} \mathrm{e}^{-c y} \mathrm{e}^{-p w} \mathrm{e}^{-q x} \mathrm{e}^{-r y}\left(\int_{0}^{\infty} \mathrm{e}^{-d z} \mathrm{e}^{-s z} f(w, x, y, z) \mathrm{d} z\right) \mathrm{d} w \mathrm{~d} x \mathrm{~d} y .
\end{aligned}
$$

The inner integral with respect to $z$ in Equation (5.2) can be solved after proper substitution as

$$
\int_{0}^{\infty} \mathrm{e}^{-d z} \mathrm{e}^{-s z} f(w, x, y, z) \mathrm{d} z=\int_{0}^{\infty} \mathrm{e}^{-(s+d) z} f(w, x, y, z) \mathrm{d} z=F^{z}(w, x, y, s+d) .
$$

Equation (5.2) becomes

$$
\begin{aligned}
& L_{w x y z}\left[\mathrm{e}^{-a w} \mathrm{e}^{-b x} \mathrm{e}^{-c y} \mathrm{e}^{-d z} f(w, x, y, z)\right](p, q, r, s) \\
& =\iiint_{0}^{\infty} \mathrm{e}^{-a w} \mathrm{e}^{-b x} \mathrm{e}^{-c y} \mathrm{e}^{-p w} \mathrm{e}^{-q x} \mathrm{e}^{-r y} F^{z}(w, x, y, s+d) \mathrm{d} w \mathrm{~d} x \mathrm{~d} y \\
& =\iint_{0}^{\infty} \mathrm{e}^{-a w} \mathrm{e}^{-b x} \mathrm{e}^{-p w} \mathrm{e}^{-q x}\left(\int_{0}^{\infty} \mathrm{e}^{-c y} \mathrm{e}^{-r y} F^{z}(w, x, y, s+d) \mathrm{d} y\right) \mathrm{d} w \mathrm{~d} x .
\end{aligned}
$$

The inner integral with respect to $y$ in Equation (5.4) can be solved after proper substitution as

$$
\int_{0}^{\infty} \mathrm{e}^{-c y} \mathrm{e}^{-r y} F^{z}(w, x, y, s+d) \mathrm{d} y=\int_{0}^{\infty} \mathrm{e}^{-(c+r) y} F^{z}(w, x, y, s+d) \mathrm{d} y=F^{y z}(w, x, c+r, s+d) .
$$

After substituting the value from Equation (5.5) in Equation (5.4), we can get

$$
L_{w x y z}\left[\mathrm{e}^{-a w} \mathrm{e}^{-b x} \mathrm{e}^{-c y} \mathrm{e}^{-d z} f(w, x, y, z)\right](p, q, r, s)=\iint_{0}^{\infty} \mathrm{e}^{-a w} \mathrm{e}^{-b x} \mathrm{e}^{-p w} \mathrm{e}^{-q x} F^{y z}(w, x, r+c, s+d) \mathrm{d} w \mathrm{~d} x .
$$

On the similar lines, integrating Equation (5.6) twice accordingly, we can obtain required result Equation 
(5.1).

\section{Property (2)}

$$
\frac{1}{\alpha \beta \gamma \delta} F^{w x y z}\left(\frac{p}{\alpha}, \frac{q}{\beta}, \frac{r}{\gamma}, \frac{s}{\delta}\right)=L_{w x y z}[f(\alpha w, \beta x, \gamma y, \delta z)](p, q, r, s) .
$$

Proof: By definition (2.1) of quadruple Laplace transform right hand side of (5.7) can be solved as

$$
\begin{aligned}
& L_{w x y z}[f(\alpha w, \beta x, \gamma y, \delta z)](p, q, r, s) \\
& =\iiint \int_{0}^{\infty} \mathrm{e}^{-p w} \mathrm{e}^{-q x} \mathrm{e}^{-r y} \mathrm{e}^{-s z} f(\alpha w, \beta x, \gamma y, \delta z) \mathrm{d} w \mathrm{~d} x \mathrm{~d} y \mathrm{~d} z \\
& =\iiint_{0}^{\infty} \mathrm{e}^{-p w} \mathrm{e}^{-q x} \mathrm{e}^{-r y}\left(\int_{0}^{\infty} \mathrm{e}^{-s z} f(\alpha w, \beta x, \gamma y, \delta z) \mathrm{d} z\right) \mathrm{d} w \mathrm{~d} x \mathrm{~d} y .
\end{aligned}
$$

The inner integral with respect to $z$ can be solved, after proper substitution as

$$
\int_{0}^{\infty} \mathrm{e}^{-s z} f(\alpha w, \beta x, \gamma y, \delta z) \mathrm{d} z=\frac{1}{\delta} F^{z}\left(w, x, y, \frac{s}{\delta}\right) .
$$

On the similar lines, integrating Equation (5.9) with respect to $y, x, w$ after proper substitution, we can obtain required result Equation (5.7).

Property (3)

$$
(-1)^{m+n+\mu+\nu} \frac{\partial^{m+n+\mu+\nu}}{\partial p^{m} \partial q^{n} \partial r^{\mu} \partial s^{v}} F^{w x y z}(p, q, r, s)=L_{w x y z}\left[w^{m} x^{n} y^{\mu} z^{v} f(w, x, y, z)\right] .
$$

Proof: By definition (2.1) of quadruple Laplace transform left hand side of (5.10) can be solved as

$$
\begin{aligned}
& (-1)^{m+n+\mu+v} \frac{\partial^{m+n+\mu+\nu}}{\partial p^{m} \partial q^{n} \partial r^{\mu} \partial s^{v}} F^{w x y z}(p, q, r, s) \\
& =(-1)^{m+n+\mu+v} \frac{\partial^{m+n+\mu+\nu}}{\partial p^{m} \partial q^{n} \partial r^{\mu} \partial s^{v}} \iiint \int_{0}^{\infty} \mathrm{e}^{-p w} \mathrm{e}^{-q x} \mathrm{e}^{-r y} \mathrm{e}^{-s z} f(w, x, y, z) \mathrm{d} w \mathrm{~d} x \mathrm{~d} y \mathrm{~d} z \\
& =(-1)^{m+n+\mu+v} \frac{\partial^{m+n+\mu}}{\partial p^{m} \partial q^{n} \partial r^{\mu}} \iiint_{0}^{\infty} \mathrm{e}^{-p w} \mathrm{e}^{-q x} \mathrm{e}^{-r y}\left(\int_{0}^{\infty} \frac{\partial^{v}}{\partial s^{v}} \mathrm{e}^{-s z} f(w, x, y, z) \mathrm{d} z\right) \mathrm{d} w \mathrm{~d} x \mathrm{~d} y .
\end{aligned}
$$

The inner integral with respect to $z$ can be solved by parts and gives

$$
\int_{0}^{\infty} \frac{\partial^{v}}{\partial s^{v}} \mathrm{e}^{-s z} f(w, x, y, z) \mathrm{d} z=(-1)^{v} L_{z}\left\{z^{v} f(w, x, y, z)\right\}
$$

Equation (5.11) becomes

$$
\begin{aligned}
& (-1)^{m+n+\mu+v} \frac{\partial^{m+n+\mu+v}}{\partial p^{m} \partial q^{n} \partial r^{\mu} \partial s^{v}} F^{w x y z}(p, q, r, s) \\
& =(-1)^{m+n+\mu+v} \frac{\partial^{m+n+\mu}}{\partial p^{m} \partial q^{n} \partial r^{\mu}} \iiint_{0}^{\infty} \mathrm{e}^{-p w} \mathrm{e}^{-q x} \mathrm{e}^{-r y}\left((-1)^{v} L_{z}\left\{z^{v} f(w, x, y, z)\right\}\right) \mathrm{d} w \mathrm{~d} x \mathrm{~d} y \\
& =(-1)^{m+n+\mu} \frac{\partial^{m+n+\mu}}{\partial p^{m} \partial q^{n} \partial r^{\mu}} \iiint_{0}^{\infty} \mathrm{e}^{-p w} \mathrm{e}^{-q x} \mathrm{e}^{-r y}\left(L_{z}\left\{z^{v} f(w, x, y, z)\right\}\right) \mathrm{d} w \mathrm{~d} x \mathrm{~d} y .
\end{aligned}
$$

On the similar lines, integrating Equation (5.13) accordingly with respect to $y, x$, w, we can obtain required result Equation (5.10).

Theorem 3. A function $f(w, x, y, t)$ which is continuous on $[0, \infty)$ and satisfies the growth condition (3.1) can be recovered from only $F^{w x y z}(p, q, r, s)$ as

$$
f(w, x, y, z)=\lim _{n_{1}, n_{2}, n_{3}, n_{4} \rightarrow \infty} \frac{(-1)^{n_{1}+n_{2}+n_{3}+n_{4}}}{n_{1} ! n_{2} ! n_{3} ! n_{4} !}\left(\frac{n_{1}}{w}\right)^{n_{1}+1}\left(\frac{n_{2}}{x}\right)^{n_{2}+1}\left(\frac{n_{3}}{y}\right)^{n_{3}+1}\left(\frac{n_{4}}{z}\right)^{n_{4}+1} \frac{\partial^{n_{1}+n_{2}+n_{3}+n_{4}}}{\partial n_{1} \partial n_{2} \partial n_{3} \partial n_{4}}\left[\frac{n_{1}}{w}, \frac{n_{2}}{x}, \frac{n_{3}}{y}, \frac{n_{4}}{z}\right] .
$$

To check the efficiency of the theorem, we consider the following example. 
Let $f(w, x, y, z) \mathrm{e}^{-a w-b x-c y-d z}$ for which Laplace transform can easily be found as

$$
F^{w \times y z}(p, q, r, s)=\frac{1}{(p+a)(q+b)(r+c)(s+d)} .
$$

Taking higher order mixed derivatives of Equation (5.14), leads

$$
\frac{\partial^{n_{1}+n_{2}+n_{3}+n_{4}}}{\partial n_{1} \partial n_{2} \partial n_{3} \partial n_{4}} F^{w x y z}(p, q, r, s)=\frac{n_{1} ! n_{2} ! n_{3} ! n_{4} !(-1)^{n_{1}+n_{2}+n_{3}+n_{4}}}{(p+a)^{n_{1}+1}(q+b)^{n_{2}+1}(r+c)^{n_{3}+1}(s+d)^{n_{4}+1}} .
$$

Using (5.14) and theorem 5.1, yields

$$
\begin{aligned}
f(w, x, y, z) & =\lim _{n_{1} n_{2}, n_{3}, n_{4} \rightarrow \infty}\left(\frac{n_{1}}{w}\right)^{n_{1}+1}\left(\frac{n_{2}}{x}\right)^{n_{2}+1}\left(\frac{n_{3}}{y}\right)^{n_{3}+1}\left(\frac{n_{4}}{z}\right)^{n_{4}+1}\left(a+\frac{n_{1}}{w}\right)^{-n_{1}-1}\left(b+\frac{n_{2}}{x}\right)^{-n_{2}-1}\left(c+\frac{n_{3}}{y}\right)^{-n_{3}-1}\left(d+\frac{n_{4}}{z}\right)^{-n_{4}-1} \\
& =\lim _{n_{1} n_{2}, n_{3}, n_{4} \rightarrow \infty}\left(1+\frac{a w}{n_{1}}\right)^{-n_{1}-1}\left(1+\frac{b x}{n_{2}}\right)^{-n_{2}-1}\left(1+\frac{c y}{n_{3}}\right)^{-n_{3}-1}\left(1+\frac{d z}{n_{4}}\right)^{-n_{4}-1} .
\end{aligned}
$$

Using the application of logarithm and the L'Hospital's rule on the previous expression reveals

$$
\begin{aligned}
& \ln f(w, x, y, z)=-a w-b x-c y-d z, \\
& f(w, x, y, z)=\mathrm{e}^{-a w-b x-c y-d z} .
\end{aligned}
$$

\section{Numerical Examples}

To illustrate the applicability and effectiveness of our method, some examples are constructed in this section.

Example 4.1. Consider the following fourth order partial differential equation

$$
\begin{aligned}
& \frac{\partial^{4}}{\partial w \partial x \partial y \partial z} u(w, x, y, z)-u(w, x, y, z)=0, \\
& u(0, x, y, z)=\mathrm{e}^{x+y+z}, \quad u(w, 0, y, z)=\mathrm{e}^{w+y+z}, \\
& u(w, x, 0, z)=\mathrm{e}^{w+x+z}, \quad u(w, x, y, 0)=\mathrm{e}^{w+x+y} .
\end{aligned}
$$

Applying quadruple Laplace transform $L_{w x y z}$ on both sides of Equation (6.1), gives

$$
(p q r s-1) U(p, q, r, s)-G(p, q, r, s)=0 \text {, }
$$

where

$$
\begin{aligned}
G(p, q, r, s)= & p q r F^{w x y}(p, q, r, 0)+p q s F^{w z z}(p, q, 0, s)+p r s F^{w y z}(p, 0, r, s)+q r s F^{x y z}(0, q, r, s) \\
& -p q F^{w x}(p, q, 0,0)-p r F^{w y}(p, 0, r, 0)-p s F^{w z}(p, 0,0, s)-q r F^{x y}(0, q, r, 0) \\
& -q s F^{x z}(0, q, 0, s)-r s F^{y z}(0,0, r, s)+p F^{w}(p, 0,0,0)+q F^{x}(0, q, 0,0) \\
& +r F^{y}(0,0, r, 0)+s F^{z}(0,0,0, s)-f(0,0,0,0) \\
= & \frac{(p q r s-1)}{(p-1)(q-1)(r-1)(s-1)} .
\end{aligned}
$$

After substituting the value, Equation (6.1) becomes

$$
U(p, q, r, s)=\frac{1}{(p-1)(q-1)(r-1)(s-1)} .
$$

Applying the quadruple inverse Laplace transform on (6.3)

$$
u(w, x, y, z)=L_{w x y z}^{-1}\left[\frac{1}{(p-1)(q-1)(r-1)(s-1)}\right]=\mathrm{e}^{w+x+y+z} .
$$


Example 4.2. Consider the following three-dimensional diffusion equations [9]

$$
\begin{aligned}
& \frac{\partial^{2} u}{\partial x^{2}}+\frac{\partial^{2} u}{\partial y^{2}}+\frac{\partial^{2} u}{\partial z^{2}}=\frac{\partial u}{\partial t}, \\
& u(0, y, z, t)=\mathrm{e}^{y+z+3 t}, \quad u(1, y, z, t)=\mathrm{e}^{1+y+z+3 t}, \\
& u(x, 0, z, t)=\mathrm{e}^{x+z+3 t}, \quad u(x, 1, z, t)=\mathrm{e}^{x+1+z+3 t}, \\
& u(x, y, 0, t)=\mathrm{e}^{x+y+3 t}, \quad u(x, y, 1, t)=\mathrm{e}^{x+y+1+3 t}, \\
& u(x, y, z, 0)=\mathrm{e}^{x+y+z} .
\end{aligned}
$$

Applying quadruple Laplace transform $L_{w x y z}$ on both sides of Equation (6.5), gives

$$
\left(p^{2}+q^{2}+r^{2}-s\right) U(p, q, r, s)=G(p, q, r, s),
$$

where

$$
\begin{aligned}
G(p, q, r, s)= & p F^{y z t}(0, q, r, s)+F_{x}^{y z t}(0, q, r, s)+q F^{x z t}(p, 0, r, s)+F_{y}^{x z t}(p, 0, r, s) \\
& +r F^{x y t}(p, q, 0, s)+F_{z}^{x y t}(p, q, 0, s)-F^{x y z}(p, q, r, 0) \\
= & \frac{\left(p^{2}+q^{2}+r^{2}-s\right)}{(p-1)(q-1)(r-1)(s-3)} .
\end{aligned}
$$

After substituting the value of $G(p, q, r, s)$, Equation (6.5) becomes

$$
U(p, q, r, s)=\frac{1}{(p-1)(q-1)(r-1)(s-3)} .
$$

Applying the quadruple inverse Laplace transform on (6.7)

$$
u(x, y, z, t)=L_{x y z t}^{-1}\left[\frac{1}{(p-1)(q-1)(r-1)(s-3)}\right]=\mathrm{e}^{x+y+z+3 t} .
$$

Example 4.3. Consider the following non-homogeneous fourth order partial differential equation

$$
\left.\begin{array}{cc}
\frac{\partial^{4}}{\partial w \partial x \partial y \partial z} u(w, x, y, z)+u(w, x, y, z)=5 \mathrm{e}^{w-2 x+y-2 z}, \\
u(0, x, y, z)=\mathrm{e}^{-2 x+y-2 z}, & u(w, 0, y, z)=\mathrm{e}^{w+y-2 z}, \\
u(w, x, 0, z)=\mathrm{e}^{w-2 x-2 z}, & u(w, x, y, 0)=\mathrm{e}^{w-2 x+y} .
\end{array}\right\}
$$

Applying quadruple Laplace transform $L_{w x y z}$ on both sides of Equation (6.9), gives

$$
(p q r s+1) U(p, q, r, s)=\frac{5}{(p-1)(q+2)(r-1)(s+2)}+G(p, q, r, s),
$$

where

$$
\begin{aligned}
G(p, q, r, s)= & p q r F^{w x y}(p, q, r, 0)+p q s F^{w z z}(p, q, 0, s)+p r s F^{w y z}(p, 0, r, s)+q r s F^{x y z}(0, q, r, s) \\
& -p q F^{w x}(p, q, 0,0)-p r F^{w y}(p, 0, r, 0)-p s F^{w z}(p, 0,0, s)-q r F^{x y}(0, q, r, 0) \\
& -q s F^{x z}(0, q, 0, s)-r s F^{y z}(0,0, r, s)+p F^{w}(p, 0,0,0)+q F^{x}(0, q, 0,0) \\
& +r F^{y}(0,0, r, 0)+s F^{z}(0,0,0, s)-f(0,0,0,0) \\
= & \frac{(p q r s-4)}{(p-1)(q+2)(r-1)(s+2)} .
\end{aligned}
$$

After substituting the value of $G(p, q, r, s)$, Equation (6.9) becomes 


$$
U(p, q, r, s)=\frac{1}{(p-1)(q+2)(r-1)(s+2)} .
$$

Applying the quadruple inverse transform on (11)

$$
u(w, x, y, z)=L_{w x y z}^{-1}\left[\frac{1}{(p-1)(q+2)(r-1)(s+2)}\right]=\mathrm{e}^{w-2 x+y-2 z} .
$$

Example 4.4. Consider the following non-homogeneous three-dimensional diffusion equation

$$
\begin{aligned}
& \frac{\partial^{2} u}{\partial x^{2}}+\frac{\partial^{2} u}{\partial y^{2}}+\frac{\partial^{2} u}{\partial z^{2}}-\frac{\partial u}{\partial t}=4 \mathrm{e}^{x-y+z-t}, \\
& u(0, y, z, t)=\mathrm{e}^{-y+z-t}, \quad u(1, y, z, t)=\mathrm{e}^{1-y+z-t}, \\
& u(x, 0, z, t)=\mathrm{e}^{x+z-t}, \quad u(x, 1, z, t)=-\mathrm{e}^{x-1+z-t}, \\
& u(x, y, 0, t)=\mathrm{e}^{x-y+z}, \quad u(x, y, 1, t)=\mathrm{e}^{x-y+1-t}, \\
& u(x, y, z, 0)=\mathrm{e}^{x-y+z} .
\end{aligned}
$$

Applying quadruple Laplace transform $L_{w x y z}$ on both sides of Equation (6.13), gives

$$
\left(p^{2}+q^{2}+r^{2}-s\right) U(p, q, r, s)=G(p, q, r, s)+\frac{4}{(p-1)(q+1)(r-1)(s+1)},
$$

where

$$
\begin{aligned}
G(p, q, r, s)= & p F^{y z t}(0, q, r, s)+F_{x}^{y z t}(0, q, r, s)+q F^{x z t}(p, 0, r, s)+F_{y}^{x z t}(p, 0, r, s) \\
& +r F^{x y t}(p, q, 0, s)+F_{z}^{x y t}(p, q, 0, s)+F^{x y z}(p, q, r, 0) . \\
= & \frac{p^{2}+q^{2}+r^{2}-s-4}{(p-1)(q+1)(r-1)(s+1)} .
\end{aligned}
$$

After substituting the value $G(p, q, r, s)$, Equation (6.13) becomes

$$
U(p, q, r, s)=\frac{1}{(p-1)(q+1)(r-1)(s+1)} .
$$

Applying the quadruple inverse transform on (6.15)

$$
u(x, y, z, t)=L_{x y z t}^{-1}\left[\frac{1}{(p-1)(q+1)(r-1)(s+1)}\right]=\mathrm{e}^{x-y+z-t} .
$$

\section{Conclusion}

In this paper, we extend the work of [4] [5] to quadruple Laplace transform. Existence and uniqueness of the quadruple transform are also discussed in this work. Some properties, theorems using the new quadruple Laplace transform and a table in which quadruple Laplace transform applied on some functions have also been presented. It is analyzed that our proposed method is well suited for use in partial differential equation involving four variables. Therefore, the present method is an accurate and reliable technique for the partial differential equations.

\section{References}

[1] Kurnaz, A. and Oturanç, G. (2005) N-Dimensional Differential Transformation Method for Solving Partial Differential Equations. International Journal of Computer Mathematics, 167, 369-380. http://dx.doi.org/10.1080/0020716042000301725

[2] Evans, D.J., Ergu, M. and Bulut, H. (2003) Variational Iteration Method-A Kind of Nonlinear Analytical Technique: Some Examples. International Journal of Computer Mathematics, 80, 1189-1198. http://dx.doi.org/10.1080/00207160310001597161 
[3] Inc, M. and Evans, D.J. (2004) An Efficient Approach to Approximate Solutions of Eighth-Order Boundary-Value Problems. International Journal of Computer Mathematics, 81, 685-692. http://dx.doi.org/10.1080/0020716031000120809

[4] Atangana, A. (2013) A Note on the Triple Laplace Transform and Its Applications to Some Kind of Third-Order Differential Equation. Abstract and Applied Analysis, 2013, Article ID: 769102. http://dx.doi.org/10.1155/2013/769102

[5] Eltayeb, H. and Kiliçman, A. (2013) A Note on Double Laplace Transform and Telegraphic Equations. Abstract and Applied Analysis, 2013, Article ID: 932578. http://dx.doi.org/10.1155/2013/932578

[6] Dahiya, R.S. and Saberi-Nadjaf, J. (1999) Theorems on N-Dimensional Laplace Transforms and Their Applications. 15th annual Conference of Applied Mathematics, Univ. of Central Oklahoma, Electronic Journal of Differential Equations, 02, 61-74.

[7] Aghili, A. and Moghaddam, B.S. (2008) Laplace Transform Pairs of n-Dimensions and Second Order Linear Partial Differential Equations with Constant Coefficients. Annales Mathematicae et Informaticae, 35, 3-10.

[8] Kiliçman, A. and Eltayeb, H. (2012) Some Remarks on the Sumudu and Laplace Transforms and Applications to Differential Equations. ISRN Applied Mathematics, 2012, Article ID: 591517. http://dx.doi.org/10.5402/2012/591517

[9] Cheniguel, A. and Reghioua, M. (2013) Ton the Numerical Solution of Three-Dimensional Diffusion Equation with an Integral Condition. Proceedings of the World Congress on Engineering and Computer Science 2013, II, 23-25.

[10] Ditkin, V.A. and Prudnikov, A.P. (1962) Operational Calculus in Two Variables and Its Applications. Pergaman Press, New York. (English Translation from Russian)

[11] Kanwal, R.P. (2013) Generalized Functions Theory and Applications. Proceedings of the World Congress on Engineering and Computer Science 2013, II, 23-25. 


\section{Appendix}

Table of quadruple Laplace transform $L_{w x y z}$ for functions of four variables.

\begin{tabular}{|c|c|}
\hline Function $f(w, x, y, z)$ & Quadruple Laplace transform $F^{\text {wxyz }}(p, q, r, s)$ \\
\hline abcd & $\frac{a b c d}{p q r s}$ \\
\hline$w x y z$ & $\frac{1}{p^{2} q^{2} r^{2} s^{2}}$ \\
\hline$w^{m} x^{n} y^{\mu} z^{v}$ & $\frac{\Gamma(1+m) \Gamma(1+n) \Gamma(1+\mu) \Gamma(1+v)}{p^{m+1} q^{n+1} r^{\mu+1} s^{v+1}}$ \\
\hline $\mathrm{e}^{-a w-b x-c y-d z}$ & $\frac{1}{(p+a)(q+b)(r+c)(s+d)}$ \\
\hline$w^{m} x^{n} y^{\mu} z^{v} \mathrm{e}^{-a w-b x-c y-d z}$ & $\frac{\Gamma(1+m) \Gamma(1+n) \Gamma(1+\mu) \Gamma(1+v)}{(p+a)(m+1)(q+b)(n+1)(r+c)(\mu+1)(s+d)(v+1)}$ \\
\hline $\cos (a w) \cos (b x) \cos (c y) \cos (d z)$ & $\frac{p q r s}{\left(p^{2}+a^{2}\right)\left(q^{2}+b^{2}\right)\left(r^{2}+c^{2}\right)\left(s^{2}+d^{2}\right)}$ \\
\hline $\sin (a w) \sin (b x) \sin (c y) \sin (d z)$ & $\frac{a b c d}{\left(p^{2}+a^{2}\right)\left(q^{2}+b^{2}\right)\left(r^{2}+c^{2}\right)\left(s^{2}+d^{2}\right)}$ \\
\hline $\cos (w+x+y+z)$ & $-\frac{p+q+r+s-p q r s}{\left(1+p^{2}\right)\left(1+q^{2}\right)\left(1+r^{2}\right)\left(1+s^{2}\right)}$ \\
\hline $\sin (w+x+y+z)$ & $\frac{p q+p r+p s+q r+q s+r s-1}{\left(1+p^{2}\right)\left(1+q^{2}\right)\left(1+r^{2}\right)\left(1+s^{2}\right)}$ \\
\hline$\sqrt{w x y z}$ & $\frac{\pi^{2}}{16 p^{2} q^{2} r^{2} s^{2}}$ \\
\hline $\cosh (a w) \cosh (b x) \cosh (c y) \cosh (d z)$ & $\frac{p q r s}{\left(p^{2}-a^{2}\right)\left(q^{2}-b^{2}\right)\left(r^{2}-c^{2}\right)\left(s^{2}-d^{2}\right)}$ \\
\hline $\sinh (a w) \sinh (b x) \sinh (c y) \sinh (d z)$ & $\frac{a b c d}{\left(p^{2}-a^{2}\right)\left(q^{2}-b^{2}\right)\left(r^{2}-c^{2}\right)\left(s^{2}-d^{2}\right)}$ \\
\hline $\mathrm{e}^{a w+b x+c y+d z} \sin (e w) \sin (f x) \sin (g y) \sin (h z)$ & $\frac{e f g h}{\left(e^{2}+(p-a)^{2}\right)\left(f^{2}+(q-b)^{2}\right)\left(g^{2}+(r-c)^{2}\right)\left(h^{2}+(s-d)^{2}\right)}$ \\
\hline $\mathrm{e}^{a w+b x+c y+d z} \cos (e w) \cos (f x) \cos (g y) \cos (h z)$ & $\frac{(p-a)(q-b)(r-c)(s-d)}{\left(e^{2}+(p-a)^{2}\right)\left(f^{2}+(q-b)^{2}\right)\left(g^{2}+(r-c)^{2}\right)\left(h^{2}+(s-d)^{2}\right)}$ \\
\hline $\mathrm{e}^{a w+b x+c y+d z} \sinh (e w) \sinh (f x) \sinh (g y) \sinh (h z)$ & $\frac{e f g h}{\left((p-a)^{2}-e^{2}\right)\left((q-b)^{2}-f^{2}\right)\left((r-c)^{2}-g^{2}\right)\left((s-d)^{2}-h^{2}\right)}$ \\
\hline $\mathrm{e}^{a w+b x+c y+d z} \cos (e w) \cos (f x) \cos (g y) \cos (h z)$ & $\frac{(p-a)(q-b)(r-c)(s-d)}{\left((p-a)^{2}-e^{2}\right)\left((q-b)^{2}-f^{2}\right)\left((r-c)^{2}-g^{2}\right)\left((s-d)^{2}-h^{2}\right)}$ \\
\hline$\frac{\sin (a w)}{w} \frac{\sin (b x)}{x} \frac{\sin (c y)}{y} \frac{\sin (d z)}{z}$ & $\tan ^{-1}\left(\frac{\sqrt{a^{2}}}{p^{2}}\right) \tan ^{-1}\left(\frac{\sqrt{b^{2}}}{q^{2}}\right) \tan ^{-1}\left(\frac{\sqrt{c^{2}}}{r^{2}}\right) \tan ^{-1}\left(\frac{\sqrt{d^{2}}}{s^{2}}\right)$ \\
\hline$w x y z \sin (a w) \sin (b x) \sin (c y) \sin (d z)$ & $\frac{16 a b c d}{\left(p^{2}+a^{2}\right)\left(q^{2}+b^{2}\right)\left(r^{2}+c^{2}\right)\left(s^{2}+d^{2}\right)^{2}}$ \\
\hline$w x y z \cos (a w) \cos (b x) \cos (c y) \cos (d z)$ & $\frac{\left(p^{2}-a^{2}\right)\left(q^{2}-b^{2}\right)\left(r^{2}-c^{2}\right)\left(s^{2}-d^{2}\right)}{\left(p^{2}+a^{2}\right)\left(q^{2}+b^{2}\right)\left(r^{2}+c^{2}\right)\left(s^{2}+d^{2}\right)^{2}}$ \\
\hline
\end{tabular}


Scientific Research Publishing (SCIRP) is one of the largest Open Access journal publishers. It is currently publishing more than 200 open access, online, peer-reviewed journals covering a wide range of academic disciplines. SCIRP serves the worldwide academic communities and contributes to the progress and application of science with its publication.

Other selected journals from SCIRP are listed as below. Submit your manuscript to us via either submit@scirp.org or Online Submission Portal.
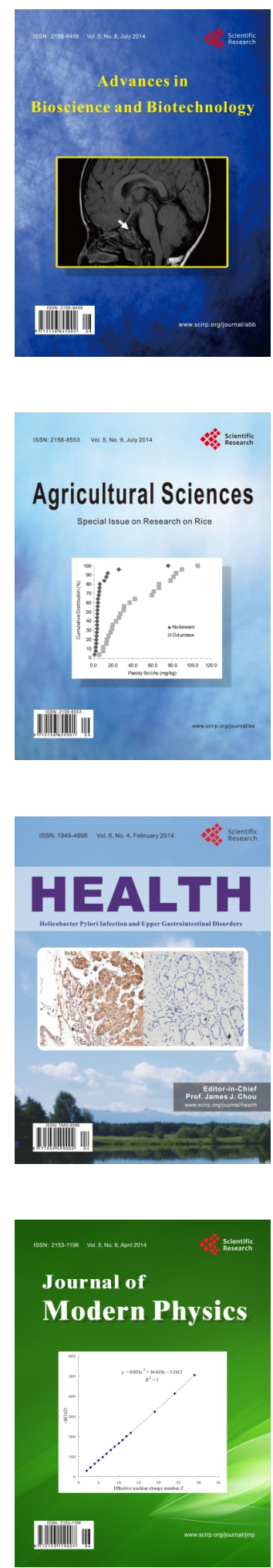
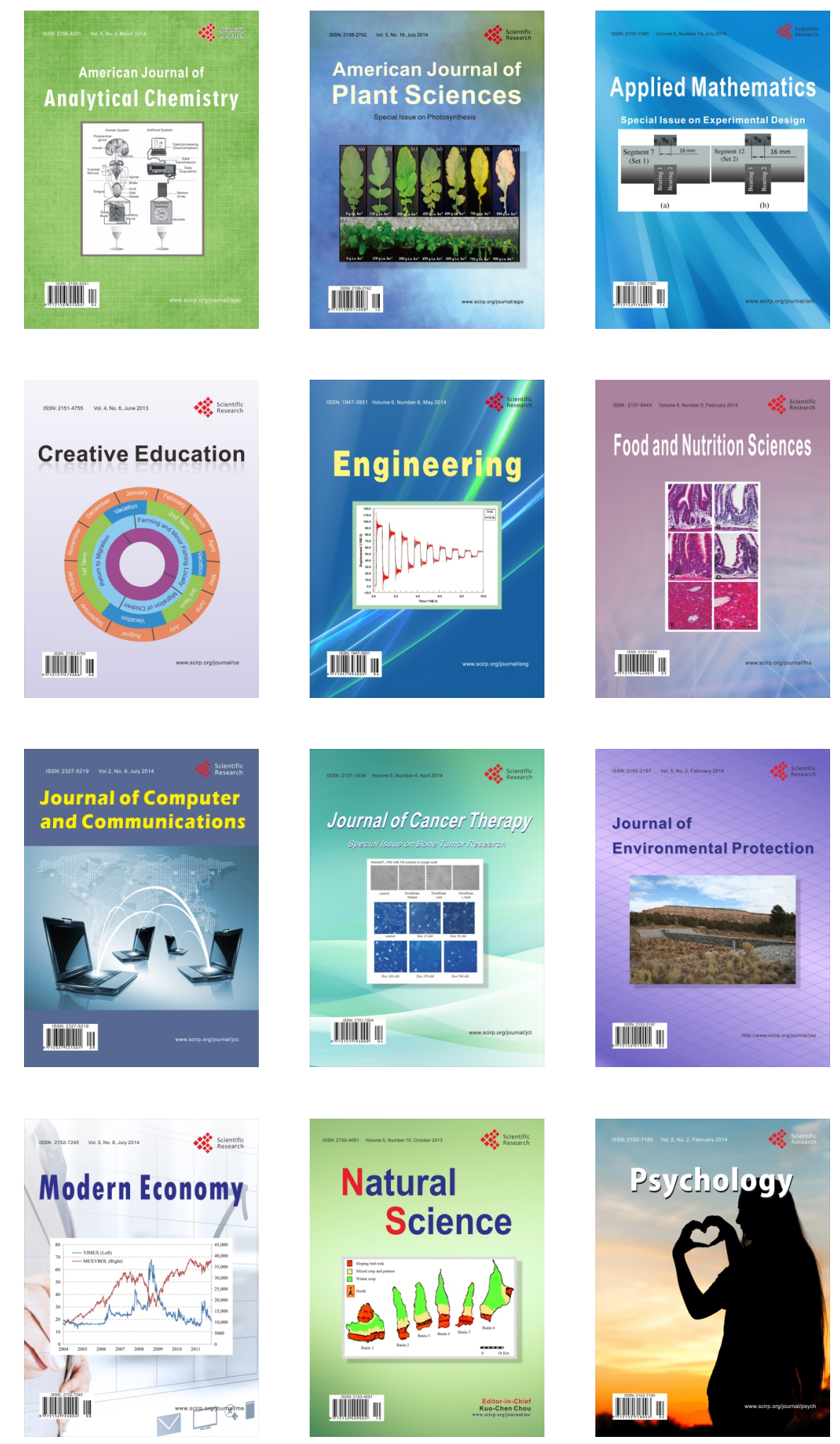